\title{
M-Theory, Torons and Confinement
}

\author{
C. Gómez and R. Hernández ${ }^{\text {a* }}$ \\ anstituto de Matemáticas y Física Fundamental, CSIC, Serrano 123, 28006 Madrid, Spain
}

\begin{abstract}
We study the decompactification limit of M-theory superpotentials for $N=1$ four dimensional supersymmetric gauge theories. These superpotentials can be interpreted as generated by toron configurations. The connection with the confinement picture in the maximal abelian gauge is discussed.
\end{abstract}

Polyakov's compact electrodynamics in three dimensions [1] constitutes a pardigmatic model for electric confinement. In the late seventies, Callan, Dashen and Gross [2] were tryig to extend the peculiar features of this model, namely the Coulomb interaction between three dimensional instantons, to four dimensions. In order to achieve this goal, they suggested a confinement mechanism based on topologically charged objects, with a fractional charge, the merons [3]. Using M-theory compactifications on an $S U(4)$ holonomy Calabi-Yau fourfold $X$, most of Polyakov's dynamics can be now derived. In particular, the instanton generated $N=2$ superpotential in three dimensions [4],

$W=\exp \left(-S_{0}-i \phi_{D}\right)$,

with $S_{0}$ the classical instanton action, and $\phi_{D}$ the dual photon field in three dimensions, can be derived using M-theory instantons [5], defined by wrapping the M-theory 5-brane on a 6 -cycle $D$ of $X$, with holomorphic aritmetic genus $\chi(D)=1$. Provided the fourfold $X$ is elliptically fibered,

$E \longrightarrow X \stackrel{\Pi}{\longrightarrow} B$

and $D$ is a vertical instanton [5], i. e., $\pi(D) \subset B$, then its effect survives the four dimensional $\epsilon \rightarrow 0$ limit, where $\epsilon$ is the Kähler class of the elliptic fiber $E$, so that we can get, in the decompactification limit, instanton contributions to the $N=1$ four dimensional superpotentials [6:7.

Super Yang-Mills Instantons. It is well known [8,9] that for pure $S U\left(N_{C}\right) N=1$ super-

\footnotetext{
*This research is partially supported by under grant AEN
} 96-1655, and by E. C. grant FMRX-CT 960012. symmetric Yang-Mills, instantons generate gluino condensates of the type

$$
<\prod_{i=1}^{N_{C}} \lambda \lambda\left(x_{i}\right)>=\text { constant } \Lambda^{3 N_{C}},
$$

with $\Lambda^{3}=\mu^{3} \exp \frac{-8 \pi^{2}}{N_{C} g^{2}}$. Correlators of type (3) are usually interpreted in terms of cluster decomposition, as products of $\langle\lambda \lambda\rangle$ condensates,

$$
<\lambda \lambda>=\text { constant } e^{2 \pi i e / N_{C}} \Lambda^{3},
$$

with $e=0, \ldots, N_{C}-1$, corresponding to the $N_{C}$ different vacua predicted by the $\operatorname{tr}(-1)^{F}$ computation [10]. The independence of (3) on the positions $x_{i}$ is due to the integration over the instanton size required by supersymmetry. In particular, the correlator $\left\langle\lambda \lambda\left(x_{1}\right) \lambda \lambda\left(x_{2}\right)>\right.$ for $S U(2)$ is saturated by instantons with size $\rho \sim\left|x_{2}-x_{1}\right|$ [8]. It is clear from (3) that instantons do not generate a superpotential for $N=1$ pure super Yang-Mills; however, it is in principle possible to define an effective superpotential [11] with a set of $N_{C}$ minima characterized by the expectation values given by (何). On the other hand, we can use the exact solution for $N=2$ pure Yang-Mills [12] and softly break to $N=1$ through the addition of a mass term. In this case, and for $S U(2)$ gauge group, the following superpotential for the monopole hypermultiplet is derived [12]:

$W=a_{D} M \tilde{M}+m u\left(a_{D}\right)$,

The two minima of (5) are now parametrized by a vacuum expectation value of the monopole field, which can be interpreted, through the dual Higgs mechanism, as the order parameter for confinement. It is, of course, tempting to identify the 
two minima of (5) with the ones described by (4i) in the $S U(2)$ case. This is an interesting dynamical issue, as the condensates (4) are in principle instanton generated, while (5) is the effective potential for the monopoles, that from the $N=1$ point of view, we should interpret as defining ' $t$ Hooft's abelian projection [13] for pure $N=1$ supersymmetric Yang-Mills theory. Of course, the interplay between confinement and the derivation of the $\langle\lambda \lambda\rangle$ condensates from the instanton amplitude (3) is contained in the cluster decomposition argument.

Torons. A different way to derive the condensate (4) using 't Hooft's torons 14 was suggested some time ago in reference 15 2. Toron configuration for $S U\left(N_{C}\right)$ gauge theories are defined using twisted boundary conditions [18] on a $T^{4}$ box of size $L$. Denoting by $n_{i j}$ the twist in the $(i, j)$-plane, the toron of Pontryagin number $\frac{1}{N_{C}}$ is defined by $n_{12}=-1, n_{34}=1$. With the definitions $m_{i} \equiv \epsilon_{i j k} n_{j k}$ and $k_{i} \equiv n_{i 4}$, the toron configuration can be interpreted as a tunneling between the states $\mid m_{3}=1, k_{3}>$ and $\mid m_{3}, k_{3}+1>$. In terms of the invariant states

$\left|e, m>\equiv \frac{1}{N_{C}^{3}} \sum_{k} e^{2 \pi i k e / N_{C}}\right| k, m>$,

the toron amplitude for $\langle\lambda \lambda\rangle$ in the $L \rightarrow \infty$ limit is given by 15

$$
\begin{array}{r}
<e, m_{3}|\lambda \lambda(x)| e, m_{3}>= \\
\frac{1}{N_{C}} \sum_{k_{3}}<k_{3}+1, m_{3}|\lambda \lambda(x)| k_{3}, m_{3}=1>= \\
\Lambda^{3} e^{2 \pi e / N_{C}},
\end{array}
$$

where now $e$ represents 't Hooft's electric flux in the 3 -direction, and can take values $e=$ $0,1, \ldots, N_{C}-1$, in agreement with result (4). Notice that the toron action is given by

$S_{\text {toron }}(e, \theta)=\frac{8 \pi^{2}}{N_{C} g^{2}}+\frac{2 \pi i e}{N_{C}}+\frac{i \theta}{N_{C}}$,

in contrast to the instanton action $\frac{8 \pi^{2}}{g^{2}}+i \theta$.

${ }^{2}$ See also references [16] and 17].

${ }^{3}$ Notice that the toron action depends on the $\theta$-parameter, and on the electric flux $e$. In fact, it is the electric flux $e$ the one that is going to play a similar dynamical role to the dual photon in the $2+1$ dimensional example.
Toron Superpotential. Let us now for a moment assume that torons are the right configuration contributing to the $\lambda \lambda$-condensates. If such is the case, taking into account that they have only two fermionic zero modes, we can in principle use this configurations to generate a superpotential for $N=1$, in such a way that their minima reproduce the correct values for the $\lambda \lambda$-condensates 19]. In the same way as for the $2+1 N=2$ example of reference 溍, we will need some information on how to define a toron dilute gas, and how to define toron correlations. Let us imagine that torons are "instanton-pieces", and that we divide the $S U\left(N_{C}\right)$ instanton into $N_{C}$ torons. Morever, we formally leave the electric flux $e$ in (8) as a free parameter; then, the superpotential, in dilute gas aproximation, for these $N_{C}$-torons would be given by

$W=\sum_{i=1}^{N_{C}} \exp \left(-S_{\text {toron }}\left(e_{i}\right)\right)$.

Now, as we impose the constraint that the $N_{C^{-}}$ torons are the pieces of an ordinary $S U\left(N_{C}\right)$ instanton, and using (8) we obtain

$$
\begin{gathered}
W=\exp \frac{-8 \pi^{2}}{N_{C} g^{2}} \sum_{i=1}^{N_{C}-1} \exp \left(\frac{-2 \pi i e_{i}}{N_{C}}\right)+ \\
\exp \frac{-8 \pi^{2}}{g^{2}} \exp \sum_{i=1}^{N_{C}-1}\left(\frac{8 \pi^{2}}{g^{2} N_{C}}+\frac{2 \pi i e_{i}}{N_{C}}\right)
\end{gathered}
$$

Notice that in (10) we have used $\theta=0$; however, no difference arises if $\theta$ is left free and common to torons and instantons. It is obvious that (10), as a superpotential for $e_{i}$ 's, possesses a set of $N_{C}$ minima for $e=0, \ldots, N_{C}-1$. It is important to stress that instantons are blind to the value of $e$, and that we fix its value at the minima of (10) only after impossing the constraint that the ordinary instanton is a "dilute gas" of $N_{C}$ torons. In fact, the previous discussion does not imply any probelm with the standard periodicity of the $\theta$-angle.

M-Theory Model. Let us now try to see how much we can learn about the origin of $\lambda \lambda$ condensates in $N=1$ using M-theory techniques. A model for $N=1$ supersymmetric Yang-Mills 
theory in four dimensions can be engineered using F-theory compactifications [6] on an elliptically fibered Calabi-Yau fourfold with $S U(4)$ holonomy. Let $C$ be a divisor in $B$ satisfying

$h^{1,0}=h^{2,0}=0$,

and such that the elliptic fiber $E$ on $C$ is singular, of ADE type in Kodaira's classification; a partially wrapped 7-brane on $C$ will define an $N=1$ ADE gauge theory in four dimensions. From now on, we will consider the simpler case of $A_{N_{C}-1}$ singularities, corresponding to $S U\left(N_{C}\right) N=1$ supersymmetric Yang-Mills in four dimensions.

From the point of view of M-theory, we can think of a compactification on an elliptically fibered Calabi-Yau, and take the $\operatorname{limit} \operatorname{Vol}(E) \rightarrow$ 0 . The resulting theory is four dimensional, with the extra dimension obtained by interpreting membranes wrapped on $E$ as Kaluza-Klein states 20]. The singular fiber $E$ of type $A_{N_{C}-1}$ has Euler characteristic

$\chi(E)=N_{C}$,

corresponding to a set of $N_{C}$ irreducible components $E_{i}$, with $E_{i} \cdot E_{i}=-2$, and intersection matrix given by the corresponding affine Dynkin diagram, $\hat{A}_{N_{C}-1}$.

As was shown in [6], we can use the irreducible components $E_{i}$ to define M-theory instantons with holomorphic genus equal one, with the corresponding divisor obtained by fibering $E_{i}$ over $C$. Each of these instantons would be characterized by the action

$S=V(C) V\left(E_{i}\right)=\frac{8 \pi^{2}}{g_{4}^{2}} \frac{\operatorname{Vol}\left(E_{i}\right)}{\operatorname{Vol}(E)}$.

Using the Kaluza-Klein 20 interpretation of membranes wrapped on $E$, we can write (13) as $\frac{R}{g_{4}^{2}} \operatorname{Vol}\left(E_{i}\right)=\frac{1}{g_{3}^{2}} \operatorname{Vol}\left(E_{i}\right)$, and to interpret $\operatorname{Vol}\left(E_{i}\right)$ as the parameters of the Coulomb branch of the $2+1$ dimensional theory. Notice that the imaginary part of $\operatorname{Vol}\left(E_{i}\right)$ represents the Wilson loop in the internal direction of effective radius $R$. The

\footnotetext{
${ }^{4}$ In order to obtain the Euler characteristic 2 should be
} assigned to each point, and -1 to each link. superpotential generated by these M-theory instantons is given by

$W=\sum_{i=1}^{N_{C}} \exp \left(-V(C) V\left(E_{i}\right)\right)$,

with the constraint

$\sum_{i=1}^{N_{C}} E_{i}=E$,

reflecting the fact that the $E_{i}$ are the $N_{C}$ components of the singular fiber $E$. In the decompactification limit $\operatorname{Vol}(E) \rightarrow 0$, the superpotential (14), with (15), becomes

$$
\begin{gathered}
W=\sum_{i=1}^{N_{C}-1} \exp \left(-V(C) V\left(E_{i}\right)\right)+ \\
\quad \gamma \exp \sum_{i=1}^{N_{C}-1}\left(V(C) V\left(E_{i}\right)\right),
\end{gathered}
$$

with $\gamma=\exp \left(\frac{-8 \pi^{2}}{g_{3}^{2} R}\right)$, and the minima corresponding to the toron action $\frac{8 \pi^{2}}{N_{C} g_{4}^{2}}+\frac{2 \pi i e}{N_{C}}$, with $e=0, \ldots, N_{C}-1$.

Thus, the partition of the M-theory divisor $E$ into irreducible components $E_{i}$ is the algebraic geometrical analog of the partition of the instanton into torons. Moreover, in the M-theory approach the 't Hooft electric flux $e$, that we have left free in (10), becomes the blow up coordinate of the singular fiber. We minimize a configuration relative to these coordinates, constrained by the decompactification condition $\operatorname{Vol}(E)=0$, in a similar way as when we constrained the values of electric flux for torons by impossing that the set of $N_{C}$ torons define an instanton.

Four Dimensions From Three Dimensions. A different approach for obtaining the superpotential 16) for $S U(2)$ was presented in reference [21]. This approach uses the AtiyahHitchin hyperkähler manifold [22] for three dimensional $N=4$ supersymmetric Yang-Mills theory. The derivation starts from the superpotential for the $N=2$ Seiberg-Witten solution [12], softly broken to $N=1$,

$W=\lambda\left(y^{2}-x^{2}(x-u)-x\right)+m u$. 
The corresponding Atiyah-Hitchin manifold is recovered upon compactification on $S^{1}$, through the definition $\frac{1}{g_{3}^{2}}=\frac{R}{g_{4}^{2}}$, in the three dimensional limit, as $m \rightarrow 0$. This can be achieved using the following change of variables [21]: $v=x-u, x=\gamma \tilde{x}$, $y=\gamma \tilde{y}$, with $\gamma=\exp \left(\frac{-8 \pi^{2}}{g_{3}^{2} R}\right)$. The superpotential (17) now becomes

$W=m\left(\frac{1}{\tilde{x}}+\gamma \tilde{x}\right)$,

which coincides with 110 for $\frac{\tilde{x}}{m} \equiv \exp \left(V_{i}\right)$.

Abelian Projection and Confinement. The crucial step in our previous discussion was to interpret the blow up parameters $V\left(E_{i}\right)$ in the $\operatorname{Vol}\left(E_{i}\right) \rightarrow 0$ limit as the electric fluxes that appear in the toron action (8). A possible way to interpret these coefficients in the $\operatorname{Vol}\left(E_{i}\right) \rightarrow 0$ limit would be as defining the abelian projection gauge 13. In the abelian projection philosophy, the gauge is determined dynamically either by the vacuum expectation value of the monopole field or, in electric variables, by the type of gauge configurations that contribute to confinement. If our interpretation of the superpotential (16) in term of torons is correct, it seems that confinement should be intimatelly related with the effective breaking of instantons into toron pieces. This effective breaking of the instanton, with a very close look to the toron liquid picture [23], is very natural from the M-theory point of view where algebraic geometry dictates the representation of the $A_{N_{C}-1}$ singular fiber $E$ in terms of $N_{C}$ irreducible components.

It would be very interesting to consider instantons in the abelian projection as sets of $N_{C}$ torons. An important role in that direction should be played by the size of the instanton. In fact, for the instanton in the singular gauge,

$A_{\mu}^{a}=2 \frac{\rho^{2}}{x^{2}+\rho^{2}} \bar{\eta}_{a \mu \nu} \frac{x_{\nu}}{x^{2}}$,

the maximal abelian gauge is obtained by minimization of the functional 2426

$G=\frac{1}{4} \int d^{4} x\left(A_{\mu}^{1}(x)^{2}+A_{\mu}^{2}(x)^{2}\right)$,

which takes the value $4 \pi \rho^{2}$.
In summary, we conclude that a fractionalization of instantons is crucial for the confinement dynamics in $N=1$ supersymmetric Yang-Mills theory, and that the M-theory description of instantons is the appropiate framework to understand the underlying dynamics.

\section{REFERENCES}

1. A. M. Polyakov, "Quark Confinement and the Topology of Gauge Groups", Nucl. Phys. B120 (1977), 429.

2. C. G. Callan, R. Dashen and D. J. Gross, "Towards a Theory of the Strong Interactions", Phys. Rev. D17 (1978), 2717.

3. V. De Alfaro, S. Fubini and G. Furlon, "A New Classical Solution of the Yang-Mills Field Equations", Phys. Lett. B65 (1976), 164.

4. I. Affleck, J. A. Harvey and E. Witten, "Instantons and Supersymmetry Breaking in $2+$ 1 Dimensions", Nucl. Phys. B206 (1982), 413.

5. E. Witten, "Non-Perturbative Superpotentials in String Theory", Nucl. Phys. B474 (1996), 343.

6. S. Katz and C. Vafa, "Geometric Engineering of $N=1$ Quantum Field Theories", hepth/9611090.

7. M. Bershadsky, A. Johansen, T. Pantev, V. Sadov and C. Vafa, "F-Theory, Geometric Engineering and $N=1$ Dualities", hepth/9612052.

8. V. Novikov, M. Shifman, A. Vainshtein and V. Zakarov, Nucl. Phys. B229 (1983), 381; Nucl. Phys. B229 (1983), 394; Nucl. Phys. B229 (1983), 407.

9. D. Amati, K. Konishi, Y. Meurice G. C. Rossi and G. Veneziano, "Non Perturbative Aspects in Supersymmetric Gauge Theories", Phys. Rep. 162 (1988), 169.

10. E. Witten, "Constrains on Supersymmetry Breaking", Nucl. Phys. B202 (1982), 253.

11. A. Kovner and M. Shifman, "Chirally Symmetric Phase of Supersymmetric Gluodynamics", hep-th/9702174.

12. N. Seiberg and E. Witten, "Electric-Magnetic Duality, Monopole Condensation and Con- 
finement in $N=2$ Supersymmetric Yang-Mills Theory", Nucl. Phys. B426 (1994), 19.

13. G. "t Hooft, "Topology of the Gauge Condition and New Confinement Phases in Non Abelian Gauge Theories", Nucl. Phys. B190 (1981), 455.

14. G. 't Hooft, "Some twisted Self-Dual Solutions for the Yang-Mills Equations on a Hypertorus", Commun. Math. Phys. 81 (1981), 267.

15. E. Cohen and C. Gómez, "Chiral Symmetry Breaking in Supersymmetric Yang-Mills", Phys. Rev. Lett. 52 (1984), 237.

16. A. R. Zhitnitsky, "Torons, Chiral Symmetry Breaking and the $U(1)$ Problem in the $\sigma$ Model and in Gauge Theories", Nucl. Phys. B340 (1990), 56.

17. H. Leutwyler and A. Smilga, "Spectrum of the Dirac Operator and Role of Winding Number in QCD", Phys. Rev. D46 (1992), 5607.

18. G. 't Hooft, "A Property of Electric and Magnetic Charges in Non Abelian Gauge Theories", Nucl. Phys. B153 (1979), 141.

19. C. Gómez and R. Hernández, "M and FTheory Instantons, $N=1$ Supersymmetry and Fractional Topological Charge", hepth/9701150.

20. S. Sethi and L. Susskind, "Rotational Invariance in the M(atrix) Formulation of Type $\mathrm{II}_{B}$ Theory", hep-th/9702101.

21. N. Seiberg and E. Witten, "Gauge Dynamics and Compactification to Three Dimensions", hep-th/9607163.

22. M. F. Atiyah and N. Hitchin, The Geometry and Dynamics of Magnetic Monopoles, Princeton University Press, 1988.

23. A. González-Arroyo. P. Martínez and A. Montero, Phys. Lett. B359 (1995), 159.

24. A. S. Kronfeld, G. Schierholz and U. Wiese, Nucl. Phys. B293 (1987), 461.

25. R. C. Brower, K. N. Orgines and C. I. Tann, "Instantons in the Maximal Abelian Gauge", Nucl. Phys. Proc. Suppl. B53 (1997), 488.

26. See, for instance, "Lattice 96", Nucl. Phys. B Proc. Suppl. 53 (1997). 